and vasculitis disease activity over the observation period ( $\mathrm{p} \leq 0.001$ for all).

Conclusions Patients who ever experience HDAS represent a distinct clinical cohort with worse longitudinal disease outcomes.

\section{PERFORMACE OF SPECIFIC (SLE-QOL) AND GENERIC (SF-36) HEALTH RELATED QUALITY OF LIFE QUESTIONNAIRES IN PATIENTS WITH SYSTEMIC LUPUS ERYTHEMATOSUS - A LONGITUDINAL STUDY}

${ }^{1}$ W Louthrenoo*, ${ }^{1} \mathrm{~N}$ Kasitanon, ${ }^{2} \mathrm{E}$ Morand, ${ }^{2} \mathrm{R}$ Kandane-Rathnayake. ${ }^{1}$ Faculty of MedicineChiang Mai University, Division of Rheumatology- Department of Internal Medicine, Chiang Mai, Thailand; ${ }^{2}$ Faculty of Medicine- Nursing and Health Sciences- Monash University, School of Clinical Sciences at Monash Health, Melbourne, Australia

\subsection{6/lupus-2017-000215.443}

Background and aims To compare specific health-related quality of life (HR-QoL) questionnaire (SLE-QoL) with a generic HRQoL questionnaire (SF-36) and to examine their sensitivity to changes defined by the global rating for change (GRC).

Methods Patients attending a single-centre lupus clinic in Thailand completed both validated SLE-QoL and SF-36 questionnaires, and rated their global change in QoL compared to previous visit using a 7-point Likert scale (GRC). Patients were grouped into either 'no change' (control group), 'deterioration' or 'improvement' categories (GRC status). Physician global assessment (PGA, 0-3) scores were collected for clinician-assessed disease activity. Associations between GRC status and SLE-QoL/SF-36 scores were examined using generalised estimating equations. Cohen's d effect sizes were estimated to compare mean changes in SLE-QoL and SF-36 scores.

Results The analysis included 248 patients with 1265 visits. Patients reported improvement in $\sim 59 \%$, deterioration in $\sim 16 \%$ and no change in QoL in $\sim 25 \%$ of visits. PGA demonstrated statistically significant $(\mathrm{p}<0.01)$, negative correlations with GRC $(r=-0.49)$, SLE-QOL $(r=-0.49)$, SF-36PCS $(r=-0.50)$ and SF-36MCS $(r=-0.36)$ scores. In contrast, SLEQoL scores correlated positively and significantly $(\mathrm{p}<0.01)$ with SF-36PCS $(r=0.56)$ and SF-36MCS $(r=0.60)$ scores. Compared to control group, mean scores of SF-36PCS, SF$36 \mathrm{MCS}$ and SLE-QoL surveys were significantly lower in the deterioration group (mean change -4.96,-3.21, and -6.51, respectively, $\mathrm{p}<0.01$ ) but SF-36PCS and SLE-QoL were significantly higher in the improvement group (mean change 1.22 and 1.35 respectively, $\mathrm{p}<0.01$ ). Cohen's d effect sizes were similar for both questionnaires.

Conclusions Both SLE-QoL and SF-36 demonstrated similar, strong associations with GRC-based deterioration or improvement.

\section{PROMOTES [PATIENT AND PHYSICIAN REPORTED OUTCOMES - MEASURES OF THE TRUE EXPERIENCE IN SLE]}

${ }^{1} \mathrm{Q}$ Luu* ${ }^{1,2} \mathrm{~V}$ Thakkar, ${ }^{1,3} \mathrm{D}$ Massasso, ${ }^{1,3,4} \mathrm{~K}$ Gibson, ${ }^{1,3,4} \mathrm{~S}$ O'Neill. 'Liverpool Hospital, Rheumatology, Liverpool BC NSW, Australia; ${ }^{2}$ University of Western Sydney, School of Medicine, Sydney, Australia; ${ }^{3}$ University of New South Wales, South Western Sydney Clinical School, Sydney, Australia; ${ }^{4}$ Ingham Institute, Rheumatology, Sydney, Australia

10.1136/lupus-2017-000215.444
Background and aims Patients with systemic lupus erythematosus (SLE) assess their disease activity differently to physicians. Prominent physician indices used to assess SLE disease activity include Systemic Lupus Erythematosus Disease Activity Index (SLEDAI) and British Isles Lupus Assessment Group (BILAG) index. We evaluated whether multidimensional health assessment questionnaire (MDHAQ), an extensively used patient reported measure, may be useful in assessing disease activity in SLE.

Methods Seventy-two consecutive patients with SLE were studied in the usual care of three rheumatologists. All patients completed an MDHAQ and the rheumatologist completed a physician global, SLEDAI and BILAG for each outpatient visit. Patients were classified as likely fibromyalgia if they scored pain $\geq 6 / 10$ and range of symptoms $\geq 16 / 60$. Scores and indices were compared using correlation and t-test.

Results Patients included 65 women and 7 men, with a total of 203 outpatient visits. In all patients, there were no correlations between patient reported outcomes and SLEDAI or BILAG. In patients without fibromyalgia, mean RAPID3, pain and patient global (PATGL) scores were significantly higher in patients with a severe BILAG class. However, none of these scores were significantly different between mild and moderate BILAG class. PATGL modestly correlated with physician global, and was usually higher than physician global.

Conclusions MDHAQ can alert the physician about the patient perception of disease activity, which is different to the physician's perspective, and is not part of the accepted SLEDAI/ BILAG assessment. These preliminary results support further study to evaluate the clinical utility of MDHAQ as a measure of SLE disease activity.

\section{\begin{tabular}{l|l}
\hline 445 & PREDICTORS OF GOOD LONG-TERM RENAL OUTCOMES
\end{tabular}}

'W Fung, ${ }^{2} \mathrm{~S}$ Su, ${ }^{3} \mathrm{Z}$ Touma, ${ }^{1} \mathrm{D}$ Gladman*. 'University of Toronto, Medicine, Toronto, Canada; ${ }^{2}$ University Health Network, Rheumatology, Toronto, Canada; ${ }^{3}$ University of Toronto, Medicine- Rheumatology, Toronto, Canada

\subsection{6/lupus-2017-000215.445}

Background and aims To determine: 1-the predictive ability of proteinuria, urinary sediment (uRBCs) and serum creatinine $(\mathrm{Cr})$ at 1 year to predict good long-term outcomes, and 2- the best proteinuria cut-off at 1 year to predict good long-term outcomes.

Methods Retrospective analysis on 1849 patients. Patients with lupus nephritis (LN) (24 hour proteinuria $[24 \mathrm{H}-\mathrm{P}]>0.5 \mathrm{~g} / \mathrm{d}$ ) with at least 7 years' follow-up were identified and baseline was defined as the onset of LN. Good renal outcome was defined as $\mathrm{Cr}<100 \mathrm{mmol} / \mathrm{L}$ and renal transplant/dialysis-free at 7 years.

ROC curves examined the predictive power of Cr, $24 \mathrm{H}-\mathrm{P}$, and $\mathrm{uRBCs}$ at 1 year post-LN diagnosis with respect to good renal outcome. AUC were analysed for: a) $24 \mathrm{H}-\mathrm{P}$ at year 1 , b) absolute change in 24H-P between year 1 and 7 , and c) percent change in 24H-P between year 1 and 7 . The proteinuria cutoff was identified by optimising sensitivity/specificity.

Results $101 \mathrm{LN}$ patients were analysed with baseline $24 \mathrm{H}-\mathrm{P}$ of $2.36 \pm 2.31 \mathrm{~g} / \mathrm{d}$. $24 \mathrm{H}-\mathrm{P}$ of $0.6 \mathrm{~g} / \mathrm{d}$ at 1 year after $\mathrm{LN}$ diagnosis best predicted good long-term renal outcome, with sensitivity 62\%/specificity 70\% (Figure 1).

AUC analysis confirmed that 24H-P at 1 year, but not absolute/percent change, is a predictor of good long-term renal outcomes (Figures 1 and 2). uRBCs did not provide any 